\title{
Sirt1 Inhibits Adipose Tissue Inflammation by Foxos/ mTOR/S6K1 Signal Pathway in Mice
}

\author{
$\operatorname{Bin} \operatorname{Li}^{1,2 *}$ \\ ${ }^{1}$ Institute of Animal Husbandry and Veterinary, Tibet Autonomous Regional Academy of Agricultural Sciences, Tibet 850000, China \\ ${ }^{2}$ State Key Laboratory of Hulless Barley and Yak Germplasm Resources and Genetic Improvement, Lhasa, Tibet 850000, China \\ *Corresponding author: Bin Li, Institute of Animal Husbandry and Veterinary, Tibet Autonomous Regional Academy of Agricultural \\ Sciences, Tibet 850000, China
}

\section{ARTICLE INFO}

Received: 蔧 July 10, 2019

Published: 蔧 July 23, 2019

Citation: Bin Li. Sirt1 Inhibits Adipose Tissue Inflammation by Foxos/mTOR/ S6K1 Signal Pathway in Mice. Biomed J Sci \& Tech Res 19(5)-2019. BJSTR. MS.ID.003371.

Keywords: Sirt; Foxo; Inflammation; Adipose; mTOR

\begin{abstract}
Sirtuin Type 1 (Sirt1) plays an important role in lipid metabolism and obesity. The overload calorie intake causes the imbalance between lipolysis and lipogenesis and leads to adipose tissue dysfunction. Moreover, adipose tissue dysfunction also attracts obesityassociated inflammation. Recent data show that Sirt1 is involved in the regulation of inflammatory progression and insulin sensitivity in macrophages and adipocytes. However, the regulatory mechanism of Sirt1 on adipose tissue inflammation remains unclear. In the current study, we found that High-Fat-Diet (HFD) reduced Sirt1 mRNA level but increased Foxo1 and Foxo3a levels in mice. Overexpressed Sirt1 increased leptin mRNA level whereas decreased the mRNA and protein levels of Foxo1, Foxo3a and inflammatory marker genes in vivo and in vitro. Moreover, we found that Foxos binds to the IL-6 promoter region to promote adipose inflammation. However, Sirt1 inhibited Foxo1 and Foxo3a by direct protein and protein interaction. Next, we found Sirt1 increased the protein levels of raptor, p-mTOR, and p-S6K1, and these results were reversed by Rapamycin addition. Despite the overexpression of Sirt1, the protein levels of Foxo1, Foxo3, and IL-6 were increased by the addition of Rapamycin. Taken together, our findings demonstrate that Sirt1 decreases adipose inflammation by directly banding with Foxos. Moreover, mTOR/S6K1 signal pathway is essential in the process of inhibiting adipose inflammation. This will promise as a novel strategy for the treatment of obesity-associated inflammation.
\end{abstract}

\section{Introduction}

Sirtuin Type 1 (Sirt1), a member of the silencing information regulator 2 (Sir2) family called sirtuins, is well known for its deacetylation regulation. Sirt1 could regulate gene silencing, cell cycle, energy homeostasis and apoptosis in adipocytes [1-3]. The overload calorie intake causes the imbalance between lipolysis and lipogenesis and leads to adipose tissue dysfunction [4]. Moreover, adipose tissue dysfunction also attracts obesity-associated inflammation [5]. Studies show Sirt1 is involved in the regulation of inflammatory progression and insulin sensitivity in macrophages and adipocytes [6,7]. Knock-out of Sirt1 in adipose tissue leads to gene expression changes which involved in obesity-related inflammation [3]. Sirt1 activator attenuates tumor necrosis factor alpha-induced insulin resistance 3T3-L1 adipocytes [8]. These suggest that Sirt1 can act as an anti-inflammation regulator. In addition, Sirt1 can deacetylate p53 and inhibit the transcriptional activity, resulting in the blockage of cell apoptosis $[9,10]$. Cinti et al. indicate Sirt1 regulates adipose tissue inflammation by controlling the gain of pro-inflammation transcription in response to inducers such as fatty acids, hypoxia, and endoplasmic reticulum stress [1113]. While numerous studies have shown Sirt1 reduces adipose inflammation, the exact regulation mechanism remains elusive.

Fox transcription factors of the 0 class (Foxos), including Foxo1, Foxo3a, Foxo4 and Foxo6 in mammals, have been reported implicated in several cellular functions, such like cell proliferation, longevity, stress resistance, metabolism. Studies reveal the negative regulated role of Foxo1 in adipocyte differentiation, and dominant negative Foxo1 enhances adipocyte differentiation by promoting cell cycle [14]. Block the function of Foxo1 in adipose tissue alters 
whole body energy homeostasis of high-fat diet induced mice, leading to the improvement of glucose and insulin tolerance and increasing energy expenditure [15,16]. Foxo1, Foxo3a, and Foxo4 are regulated by Akt signal pathway, which is essential for glucose transport, adipogenesis and white adipose cell differentiation $[17,18]$. In addition, the Foxos has been shown to mediate some effects of Sirtuins [19]. Studies show Sirt1 represses the ability of Foxo3a to activate transcription in mammal Hela cells and muscle cells. However, whether Sirt1 and Foxos physically interacted in adipocytes remains unclear.

Akt/mTOR signal pathway plays an important role in glucose production and energy metabolism [20,21]. mTOR is a highly conserved serine/threonine kinase which is phosphorylated by activated Akt in cancer cells, adipocytes and hepatocyte [22-24]. In the liver, feeding increases insulin secretion, which activates the Akt pathway in hepatocytes $[25,26]$. A recent study shows that
Akt-S473 is specifically phosphorylated by the Mammalian Target of Rapamycin (mTOR). Additionally, the disruption of macrophage mTOR signaling reduces inflammation and insulin resistance in high-fat-diet-induced mice [27]. Busch et.al suggests that Akt is one of the main upstream stimulatory kinases that modulated by Sirt1 [28]. However, whether Sirt1 could regulate obesity-related inflammation via the interaction with Akt/mTOR is remain poorly understood.

In this study, we show Sirt1 executes an anti-inflammation regulation role by interacting with Akt/Foxo1/Foxo3a. Interestingly, Foxos bind with the promoter region of IL- 6 in accentuated adipose tissue inflammation. Further investigation reveals an unexpected role of mTORC/S6K1 signals in the regulation of adipose tissue inflammation. Thus, we provide a novel regulatory mechanism of adipose inflammation by Sirt1 and Foxos interaction and Akt/ mTOR/S6K1 signal pathway is involved in this process.

\section{Results}

\section{Sirt1 Reduced Adipose Deposition and Foxos in Diet-Induced Obesity}

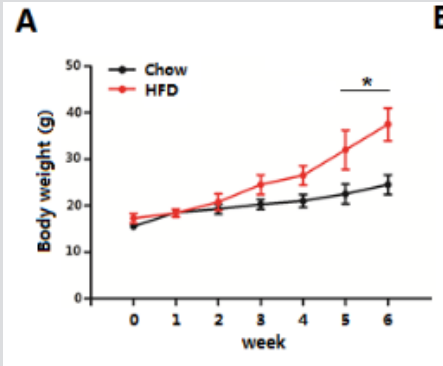

E

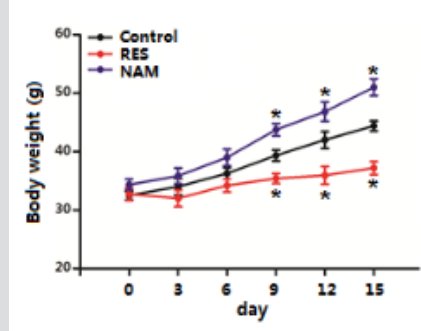

H

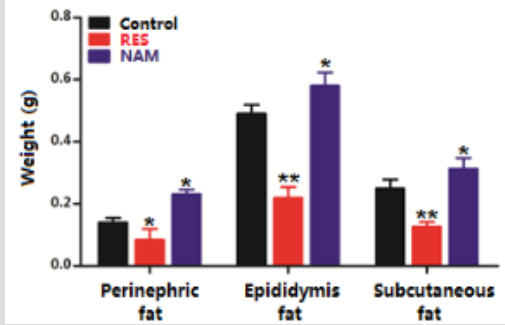

C

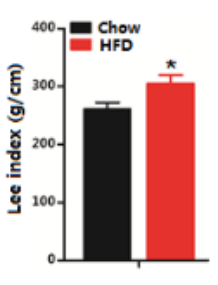

$\mathbf{F}$

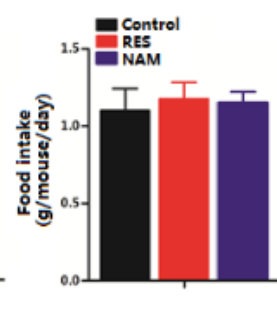

I

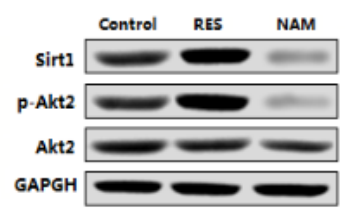

D

G
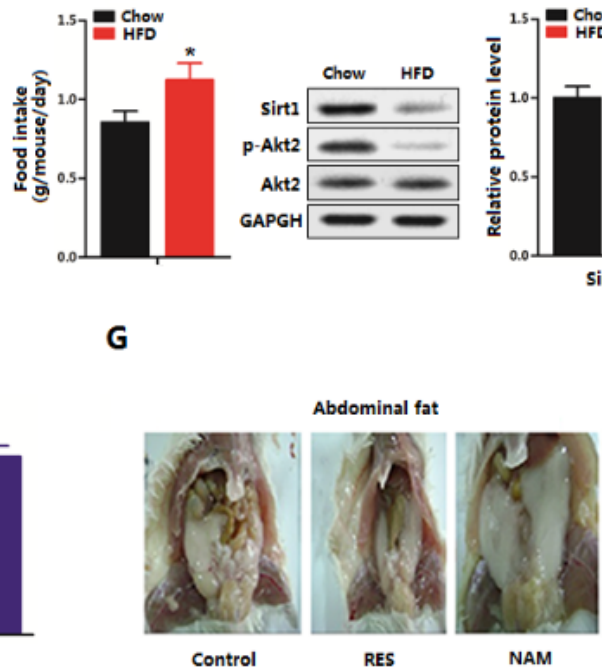

RES

NAM

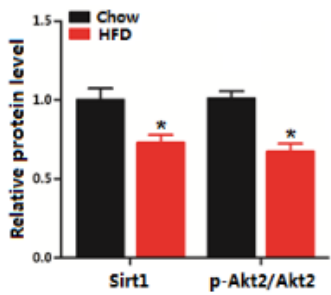

Sirt1 P-Akt2/Akt2

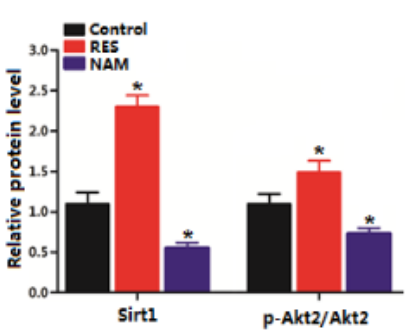

Figure 1: Sirt1 reduced adipose deposition and Foxos in diet-induced obesity. A. Body weight gain of male mice fed HFD for 7 weeks ( $n=18$ each). B. Food intake of HFD fed mice and chow diet fed mice for 7 weeks ( $=18$ each). C. mRNA levels of PPARY, IL-6, and Sirt1 after HFD fed for 7 weeks (n=18 each). D. mRNA levels of Foxo1, Foxo3a, Foxo4 and Foxo6 after HFD feeding for 7 weeks ( $n=18$ each). E. Protein levels of Sir1, Sirt2, and IL-6 with Ad-Sirt1or sh-Sirt1 tail vein injection for 5 days in HFD mice ( $\mathrm{n}=18$ each). F. mRNA levels of Foxo1 and Foxo3a with Ad-Sirt1or sh-Sirt1 tail vein injection for 5 days in HFD mice ( $n=18$ each). G. Body weight of HFD after Ad-Sirt1or sh-Sirt1 tail vein injection for 5 days ( $n=18)$. H. Food intake of HFD with Ad-Sirt1or sh-Sirt1 tail vein injection for 5 days $(n=18)$. I. Representative images of male mice abdominal fat fed of HFD with Ad-Sirt1or sh-Sirt1 tail vein injection for 5 days ( $n=18$ each). J. Visceral fat weight, epididymal fat weight and inguinal fat weight from $\mathrm{G}$ (n=18 each). Ad-Sirt1: recombinant adenovirus vector of Sirt1, sh-Sirt1: recombinant lentiviral vector of Sirt1. Values are means \pm SD. vs. control group, ${ }^{*} \mathrm{p}<0.05,{ }^{* *} \mathrm{p}<0.01$. 
To explore the effect of Sirt1 on obesity, we firstly fed six-weekold male mice on HFD for 7 weeks. Body weight and food intake were increased significantly in the HFD group (Figures 1A \& B). mRNA levels of PPAR $\gamma$ and IL-6 were increased in the HFD group compared with those in the chow diet group, while Sirt1 mRNA level was decreased (Figure 1C). Then we measured the levels of Foxo family members, Figure 1D showed Foxo1 and Foxo3a were both increased in the HFD group. The levels of Foxo4 and Foxo6 had no difference between the two treatments. After 6 weeks of HFD feeding alone, Ad-Sirt1 and sh-Sirt1 were tail vein injection for 5 days in HFD mice. The protein level of Sirt1 was increased in AdSirt1 group and decreased markedly in the sh-Sirt1 group (Figure
1E). In addition, the inflammation marker protein IL-6 was reduced after Sirt1 overexpression and along with the decreased mRNA levels of Foxo1 and Foxo3a (Figures 1E \& F). Body weight was decreased in Ad-Sirt1 group compared that in the control group, while sh-Sirt1 had the opposite effects (Figure 1G). No food intake difference was recorded among the two treatment groups (Figure 1H). Fat pads from different adipose locations (visceral, epididymal, inguinal) were all weighted less in Ad-Sirt1 treated mice compared with that in Ad-CMV treated mice and sh-Sirt1 significantly elevated fat pads weights (Figures 1I \& J). Thus, we conclude Sirt1 reduces diet-induced obesity and decreases adipose tissue inflammation respond companied with the reduction of Foxos in HFD mice.

\section{Sirt1 Reduced Foxos Expression and Played an Anti-Inflammation Role in Adipose Tissue}

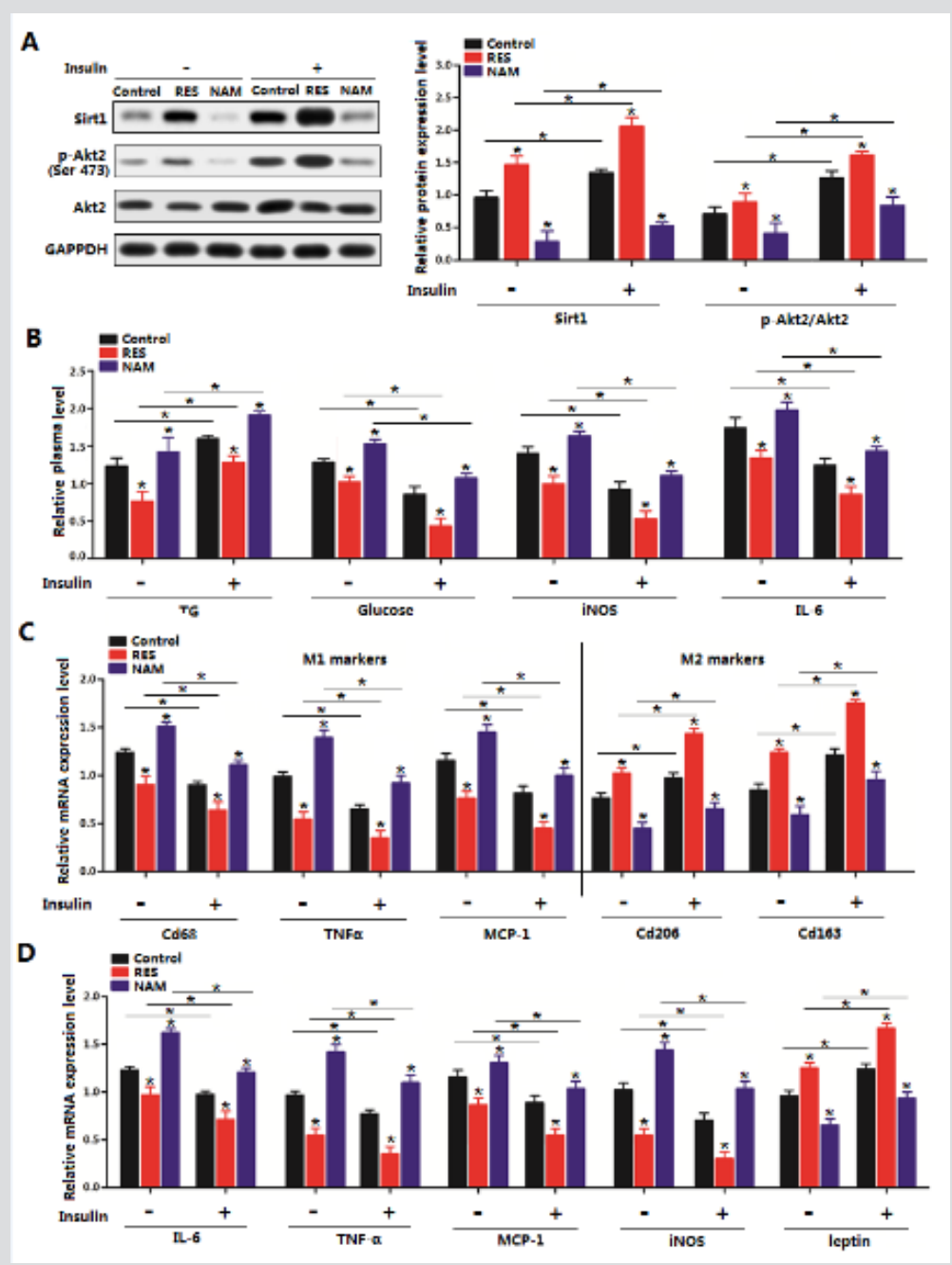

Figure 2: Sirt1 reduced Foxos level and played an anti-inflammation role in adipose tissue. A. Serum protein levels of IL-1 $\beta$, IL-6, IL-4 and IL-10 with LPS tail vein injection for 3 days $(n=12)$. B: mRNA level of Sirt1 of WAT after LPS intraperitoneal injection for 3 days $(n=12)$. C: mRNA levels of Foxo1, Foxo3a, Foxo3a and Foxo6 of WAT after LPS intraperitoneal injection for 3 days $(n=12)$. D: Serum protein levels of IL-1 $\beta$, IL-6, IL-4 and IL-10 with tail vein injection of Ad-Sirt1 or sh-Sirt1 for 5 days in mice which were pre-treated withan intraperitoneal injection of LPS for 3 days $(n=12)$. E. mRNA levels of Foxo1 and Foxo3a of WAT with tail vein injection of Ad-Sirt1 or sh-Sirt1 for 5 days and pre-treated with an intraperitoneal injection of LPS for 3 days $(n=12)$. F. mRNA levels of M1 and M2 cytokines of mice WAT macrophages with tail vein injection of Ad-Sirt1 or shSirt1 for 5 days in mice which were pre-treated with an intraperitoneal injection of LPS for 3 days $(n=12)$. G. mRNA levels of WAT inflammation cytokines with tail vein injection of Ad-Sirt1 or sh-Sirt1 for 5 days in mice which were pre-treated with an intraperitoneal injection of LPS for 3 days $(n=12)$. Ad-Sirt1: recombinant adenovirus vector of Sirt1, sh-Sirt1: recombinant lentiviral vector of Sirt1. Serum protein levels were measured by commercial ELISA kits. Values are means \pm SD. vs. control group, ${ }^{*} \mathrm{p}<0.05$ 
We next addressed whether the alternation of Sirt1 expression altered obesity-related inflammation. We first showed Lipopolysaccharide (LPS) successfully induced adipose tissue inflammation with elevated protein levels of IL-1 $\beta$, IL- 6 and reduced protein levels of IL-4 and IL-10 (Figure 2A). With LPS treatment, we also found Sirt1 had a dramatic reduction (Figure 2B). Moreover, LPS upregulated the mRNA levels Foxo1 and Foxo3a in white adipose tissue. And levels of Foxo4 and Foxo6 were comparable with LPS challenge condition (Figure 2C). To further explore the role of Sirt1 played in adipose inflammation, we measured the serum levels of IL-1 $\beta$, IL-6, IL-4 and IL-10 with Sirt1 treatment under LPS condition. Results showed that serum protein levels of IL-1 $\beta$ and IL-6 were lower in Ad-Sirt1 group and higher in sh-Sirt1 group suggesting Sirt1 effectively attenuated adipose inflammation (Figure 2D). In line with the reduction of pro-inflammation cytokines, Foxo1 and Foxo3a were downregulated after Sirt1 forced expression (Figure 2E). Consistently, overexpression of Sirt1 decreased the expression of M1 macrophage cytokines (Cd68, TNF $\alpha$, and MCP-1) which were increased by sh-Sirt1 in macrophages of WAT, whereas M2 macrophage cytokines (Cd206 and Cd163) were up-regulated significantly compared with those in control group (Figure 2F). In addition, quantitative PCR analysis further showed Sirt1 reduced the expression of IL-6, TNF $\alpha$, and MCP-1 in white adipose tissue (Figure 2G). However, the leptin level was up-regulated. Thus, our data establish that Sirt1 involves in adipose tissue inflammation by regulating Foxos expression.

\section{Sirt1 Inhibited Inflammation via Foxo1/Foxo3a/Akt Pathway in Mice Adipocyte}

We previously showed Sirt1 and Foxos family were related to adipose tissue inflammation. We first reconfirmed these results in mature adipocytes. Figures 3A-C indicated Sirt1 significantly blocked adipocyte differentiation and decreased the expression of PPAR $\gamma$ and FAS. Forced expression of Sirt1 in adipocytes significantly decreased the protein levels of IL- 6 , TNF- $\alpha$, and MCP- 1 while increased the level of leptin (Figure 3D). And in line with previous data, Sirt1 reduced the protein levels of Foxo1 and Foxo3a (Figure 3E). Further detection showed Sirt1 decreased the phosphorylation level of Akt, indicating Foxo1/Foxo3a/Akt signal pathway is involved in the regulation of Sirt1 on adipocyte inflammation (Figure 3E). And ChIP analysis demonstrated both Foxo1 and Foxo3a interacted with the promoter region of IL-6 (Figure 3E).

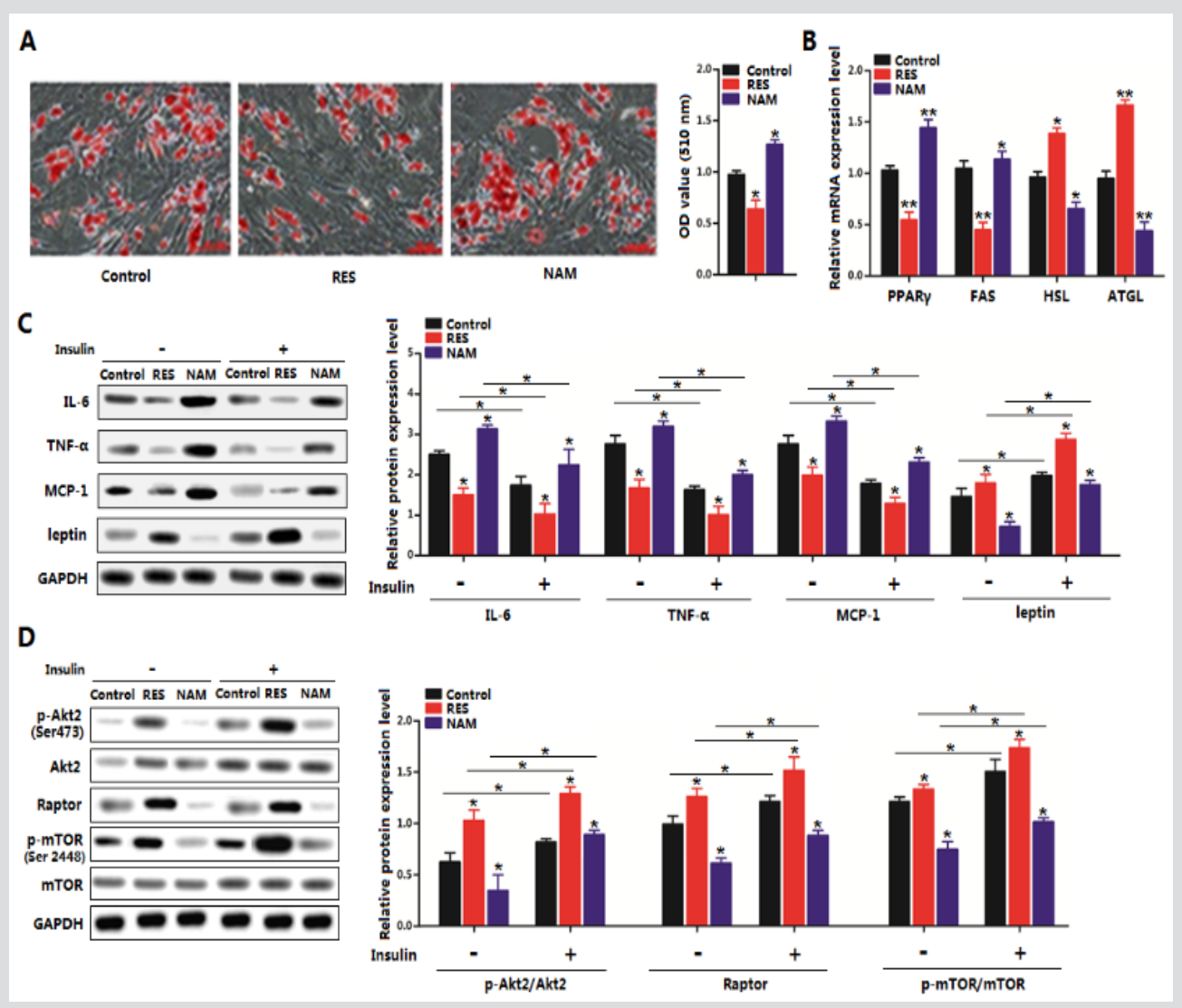

Figure 3: Sirt1 inhibited inflammation via Foxo1/Foxo3a/Akt pathway in mice adipocyte. A. Transfection efficiency detection of Ad-Sirt1 and sh-Sirt1 in mice adipocytes $(n=3)$. B. mRNA levels of FAS, HSL, ATGL, Foxo1 and Foxo3a with Ad-Sirt1 or shSirt1 transfection for $48 \mathrm{~h}(\mathrm{n}=3)$. C. TG level of primary adipocytes differentiation for $8 \mathrm{~d}$, with Ad-Sirt1or sh-Sirt1 transfection for $48 \mathrm{~h}(\mathrm{n}=3)$. D. Immunoblots of IL-6, TNF-a, MCP-1 and leptin in adipocytes transfected with Ad-Sirt1or sh-Sirt1 transfection for $48 \mathrm{~h}(\mathrm{n}=3)$. E. Immunoblots of Foxo1, Foxo3a, p-Akt, and Akt in adipocytes transfected with Ad-Sirt1 or sh-Sirt1 transfection for $48 \mathrm{~h}(\mathrm{n}=3)$. ChIP analysis of Foxo1/Foxo3a and IL-6 $(\mathrm{n}=3)$. Ad-Sirt1: recombinant adenovirus vector of Sirt1, sh-Sirt1: recombinant lentiviral vector of Sirt1. Values are means \pm SD. vs. control group, ${ }^{*} p<0.05,{ }^{* *} p<0.01$. 
Sirt1 Directly Interacted with Akt/Foxo1/Foxo3a in Obesity-Related Inflammation

To determine whether Sirt1 and Akt/Foxos interacted in adipose inflammation, we performed the protein-protein measurement. In mice adipocytes, basal Akt protein interacted with transfected Sirt1 protein (Figure 4A). Interestingly, further IP analysis showed Sirt1 also interacted with Foxo1 and Foxo3a in transfected adipocytes (Figures 4B \& C). Consider the effects of Sirt1 on adipose inflammation, we transfected adipocytes with Ad-Sirt1 (sh-Sirt1) and Ad-Foxo1 to elucidate the regulation mechanism between Sirt1 and Foxos family. Figure 4D showed co-transfected adipocytes with Ad-Sirt1 and Ad-Foxo1 increased the expression level of IL-6 compared with that in Ad-Sirt1 transfected group. While leptin was strongly reduced in the co-transfected group (Figure 4D). As expected, co-transfected adipocytes with Ad-Sirt1 and Ad-Foxo3a obtained the consistent data (Figure 4E), confirming the interaction of Sirt1 and Foxos family. Then we used the Ad-Sirt1-mutant vector and Ad-Foxo1 vector to treat cells, the result demonstrated after blocking the function of Sirt1, Foxo1 drastically increased the level of IL-6 (Figure 4F), which was the same in Ad-Sirt1-mutant and Ad-Foxo3a co-treatment group (Figure 4G). Thus, we conclude that Sirt1 executes its anti-inflammation function due to the direct reaction with Akt and Foxos family.

\section{A}
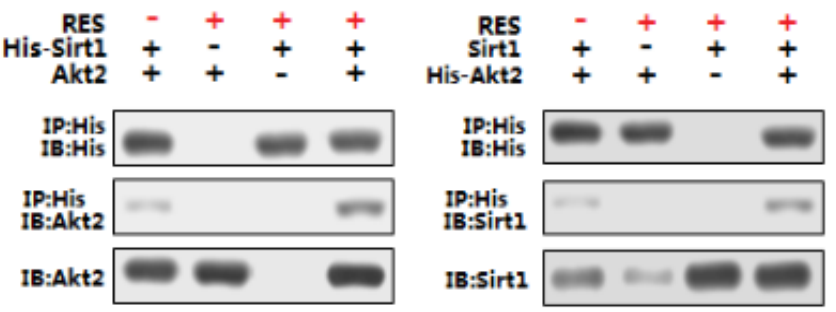

B
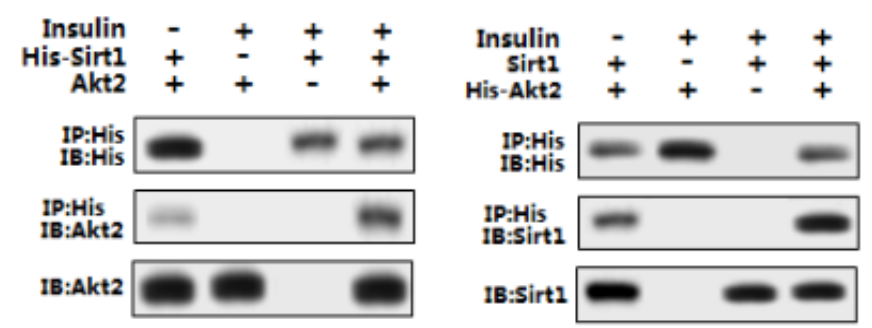

D
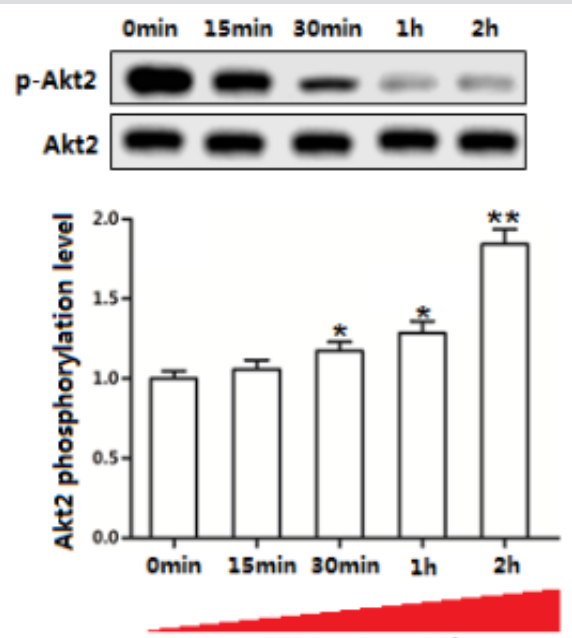

E

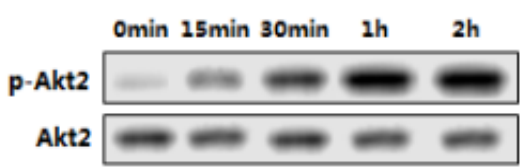

C
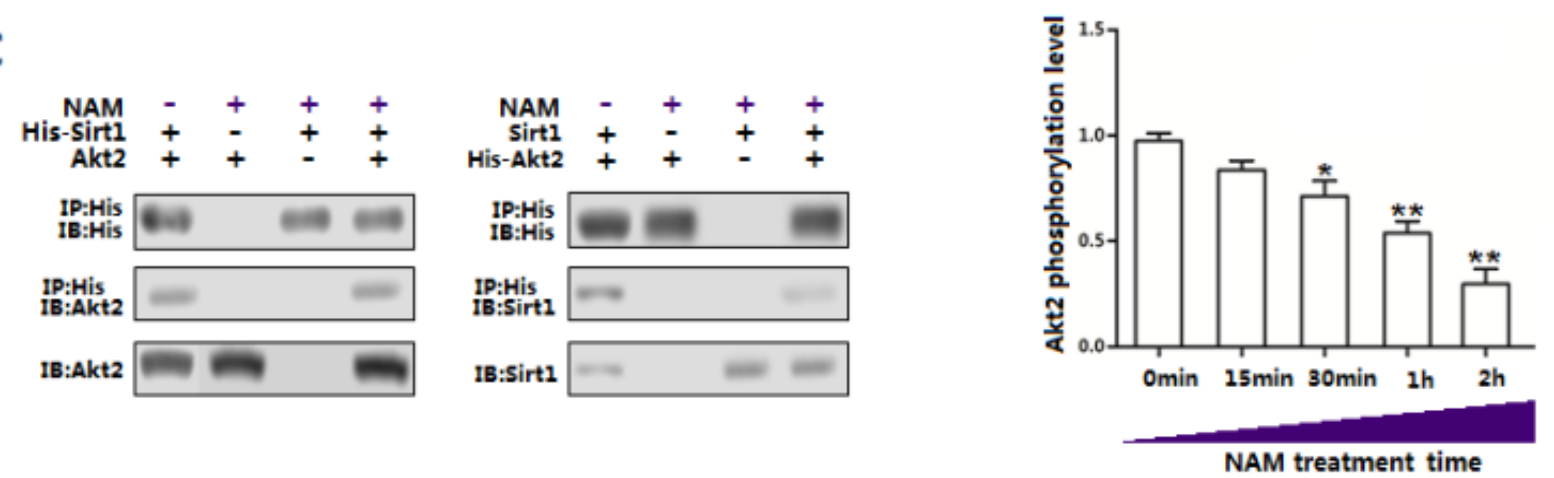

Figure 4: Sirt1 directly interacted with Akt/Foxo1/Foxo3a in obesity-related inflammation. A. Sirt1 interacts with Akt. Immunoprecipitation (IP) analysis in His-Sirt1, Akt, Sirt1 or His-Akt transfected adipocytes (n=3). B. Sirt1 interacts with Foxo1. Immunoprecipitation (IP) analysis in His-Sirt1, Foxo1, Sirt1 or His-Foxo1 transfected adipocytes (n=3). C. Sirt1 interacts with Foxo3a. Immunoprecipitation (IP) analysis in His-Sirt1, Foxo3a, Sirt1 or His-Foxo3a transfected adipocytes $(n=3)$. D. mRNA levels of IL-6 and leptin in adipocytes transfected with Ad-Sirt1, sh-Sirt1 or Ad-Foxo1 (n=3). E. mRNA levels of IL-6 and leptin in adipocytes transfected with Ad-Sirt1, sh-Sirt1 or Ad-Foxo3a $(n=3)$. F. mRNA level of IL-6 transfection with Ad-Sirt1, AdSirt1-mutant, Ad-Foxo1 or Ad-Foxo3a $(n=3)$. Ad-Sirt1: recombinant adenovirus vector of Sirt1, sh-Sirt1: recombinant lentiviral vector of Sirt1. Ad-Foxo1: recombinant adenovirus vector of Foxo1, Ad-Foxo3a: recombinant adenovirus vector of Foxo3a, His-Sirt1, and Sirt1: eukaryon expression plasmid of Sirt1. His-Foxo1 and Foxo1: eukaryon expression plasmid of Foxo1. HisFoxo3a and Foxo3a: eukaryon expression plasmid of Foxo3a. Ad-Sirt1-mutant: recombinant adenovirus vector of Sirt1 and with no function. Values are means \pm SD. vs. control group, ${ }^{*} \mathrm{p}<0.05,{ }^{* *} \mathrm{p}<0.01$. 


\section{Sirt1 Reduced Adipose Tissue Inflammation by Inhibit- ing Foxos Family}

We next characterize the Foxos family function in the regulation of Sirt1 on adipocyte inflammation. Figure 5A showed adipocytes pre-incubated with LPS for $12 \mathrm{~h}$ did not impair cell viability and induced the adipocyte inflammation successfully (Figure 5B). We found no evidence of cell apoptosis as demonstrated by the mRNA level of caspase 3 (Figure 5B). Then with the LPS challenge condition, cells co-transfected with Ad-Sirt1 and Ad-Foxo1 presented a more severe inflammation compared with that in Ad-Sirt1 alone treated group (Figure 5C). Pro-inflammation cytokines IL-6 and MCP-1 showed in Figure 5C was reduced in Sirt-1 overexpression group, while Ad-Foxo1 treatments reverse these genes expression which accentuated adipocyte inflammation. Anti-inflammation factor leptin was increased with Ad-Sirt1 transfection, however strikingly decreased after Ad-Foxo1 treating (Figure 5C). Consistently, we got the same results in Ad-Foxo3a and Ad-Sirt1 treatment group (Figure 5D). To this end, these data demonstrate Sirt1 plays an antiinflammation function through inhibiting Foxos function.

\section{mTOR/S6K1 Signal Pathway was essential for Sirt1 in the Regulation of Adipose Tissue Inflammation}

To further dissect the mechanism underlying the role of Sirt1 in the regulation of adipose inflammation we first measured the phosphorylation levels of mTORSer2448 and S6K1Thr389 with or without Sirt1 treatment. Strikingly forced expression of Sirt1 increased the level of p-mTORSer2448 and p-S6K1Thr389 while shSirt1 had the opposite effects (Figure 5A). And further addition of mTOR signaling inhibitor Rapamycin inhibited the activity of mTOR effectively, while Ad-Sirt1 transfection alleviated the inhibition of mTOR activity (Figure 5A). Additionally, we observed the activation of the mTOR signaling pathway by Sirt1 strongly reduced the levels of Foxo1, Foxo3a, and IL-6 and elevated the protein level of leptin (Figure 5B). Further addition of Rapamycin decreased the protein levels of Foxo1, Foxo3a, and IL-6 more obviously (Figure 5B). Thus, the adipose inflammation is associated with mTOR/S6K1 signaling pathway, and Sirt1 and Foxos played opposite roles in this process. Together, these data demonstrate the mTOR signal pathway is involved in Sirt1 inhibition of adipose tissue inflammation.
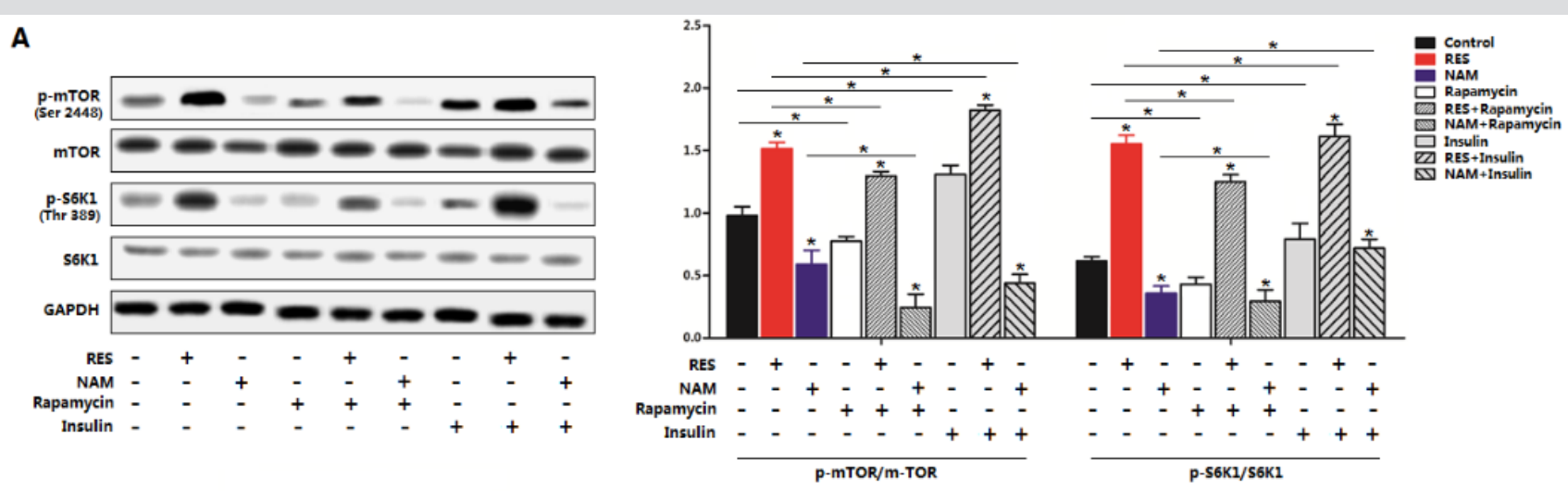

B

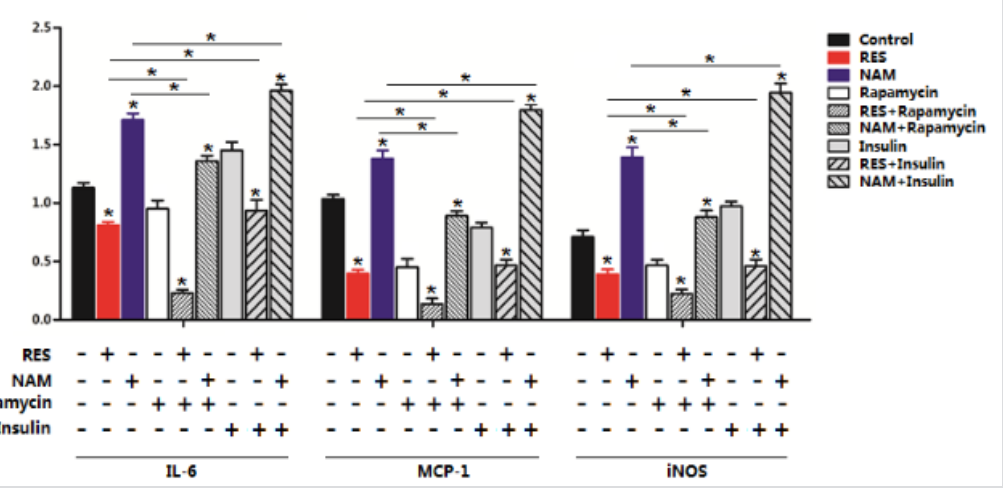

Figure 5: Sirt1 Foxos have the opposite function in adipocyte inflammation. A. Cell viability of adipocytes with LPS incubation for $12 \mathrm{~h}, 24 \mathrm{~h}$, and $48 \mathrm{~h}(\mathrm{n}=3)$. B. mRNA expression levels of IL-6, Leptin, Sirt1 and Caspase3 of adipocytes with LPS incubation for $12 \mathrm{~h}(\mathrm{n}=3)$. C. mRNA levels of IL-6, MCP-1 and leptin of adipocytes with LPS incubation for $12 \mathrm{~h}$ and pre-transfected with Ad-Sirt1, sh-Sirt1 or Ad-Foxo1 (n=3). D. mRNA levels of IL-6, MCP-1 and leptin of adipocytes with LPS incubation for 12h and pre-transfected with Ad-Sirt1, sh-Sirt1 or Ad-Foxo3a $(n=3)$. Ad-Sirt1: recombinant adenovirus vector of Sirt1, sh-Sirt1: recombinant lentiviral vector of Sirt1. Ad-Foxo1: recombinant adenovirus vector of Foxo1, Ad-Foxo3a: recombinant adenovirus vector of Foxo3a. Values are means \pm SD. vs. control group, ${ }^{*} p<0.05,{ }^{* *} p<0.01$ 


\section{Discussion}

It is now well recognized that in obesity-related diseases, chronic low-grade tissue inflammation is an important etiologic component of insulin resistance, metabolic syndrome, and type 2 diabetes $[7,29,30]$. Activation of Sirt1 leads to the improvement of glucose tolerance and insulin sensitivity [31,32]. Here our data demonstrated that HFD reduced the expression level of Sirt1 and increased the levels of Foxo1 and Foxo3a. While Foxos family members Foxo4 and Foxo6 were comparable in HFD group and control group. HFD also induced a high level of IL-6, the cytokine of inflammation. Adipose inflammation is a hot issue in decades, it represents by the secretion of pro- and anti-inflammatory cytokines such as IL- 6 and IL-10 by monocytes or macrophages. Studies have indicated interfering of Foxos in cells results in reduced expression of pro-inflammatory cytokines [33]. We hypothesized that Sirt1 and Foxos had the opposite function. Upon the direct interaction, we confirmed this effect by performing the co-transfection of Sirt1 and Foxo1/Foxo3a. Studies show that Foxos and Sirt1 can inhibit NFkappa B signaling and simultaneously protect against inflammation process [34]. Our data confirmed that Sirt1 reduced adipose tissue inflammation by inhibiting Foxo1 and Foxo3a which bind to the promoter region of IL-6 (Figure 6).

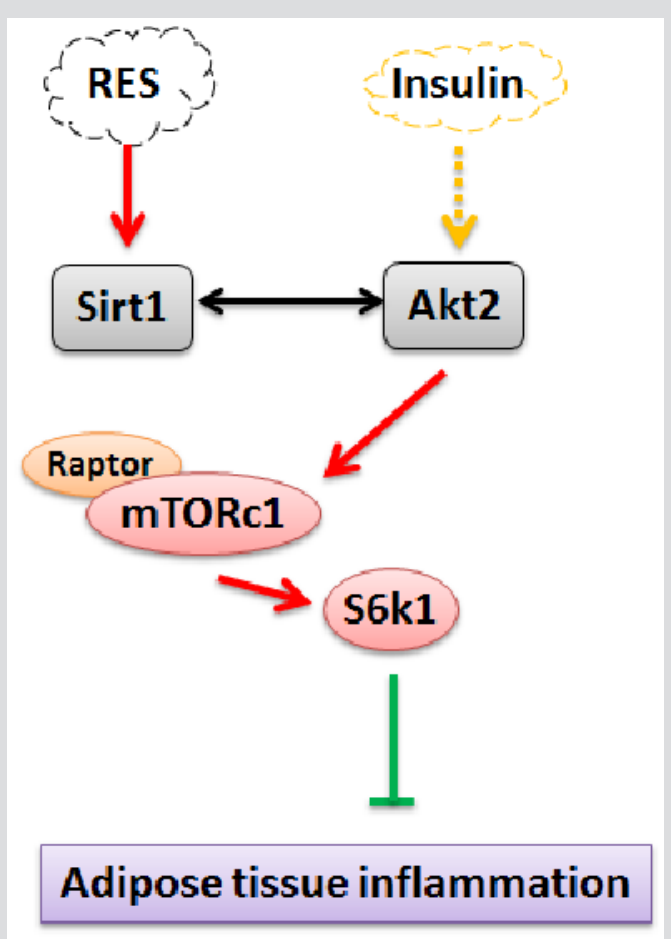

Figure 6: mTORC1/S6K1 signaling pathway is essential for the regulation of adipose inflammation of Sirt1. A. The phosphorylation levels and protein levels of mTOR and S6K1 in adipocytes pre-transfected with Ad-Sirt1 or sh-Sirt1 for 48 $\mathrm{h}$ and then treated with or without $5 \mathrm{nM}$ Rapamycin for $60 \mathrm{~min}$ or not $(\mathrm{n}=3)$. B. Immunoblots of inflammation cytokines and Foxos in adipocytes pre-transfected with Ad-Sirt1 or sh-Sirt1 for $48 \mathrm{~h}$ and then treated with or without $5 \mathrm{nM}$ Rapamycin for 60 min or not $(n=3)$. $(n=3)$. Ad-Sirt1: recombinant adenovirus vector of Sirt1, sh-Sirt1: recombinant lentiviral vector of Sirt1. Values are means $\pm \mathrm{SD}$. vs. control group, ${ }^{*} \mathrm{p}<0.05$.

Acts, the downstream effectors of insulin signal, is activated and affect metabolism, proliferation, cell survival, cell growth and inflammation $[35,36]$. Inhibiting the Akt signal pathway increased anti-inflammatory effects and decelerated the metabolic syndrome. The sirt1 level was increased in response to calorie restriction, the most consistent intervention to increase lifespan and protect against deteriorations in biological functions in many key metabolic tissues $[37,38]$. Pang et.al has been reported Sirt1 directly binding with Akt2 to inhibited adipogenesis [39]. By immunoprecipitation, we also found Sirt1 and Akt had a protein-protein interaction during adipose tissue inflammation respond. Phosphoinositide 3-Kinase (PI3K), Akt, and their downstream kinase, Mammalian Target of Rapamycin (mTOR), are implicated in insulin resistance, metabolic dysfunction, and inflammation. Here, we focused on the influence of Sirt1 on mTOR signal pathway. Our data showed Sirt1 inhibited the phosphorylation level of mTOR. This demonstrated that mTOR was involved in the regulation of adipose inflammation by Sirt1. Mammalian Target of Rapamycin (mTOR) is a serine/threonine protein kinase regulates protein synthesis [40]. Studies report that activation of mTOR plays key roles in TNF- $\alpha$-induced inflammatory cascades [41] and is also implicated in inflammation-related diseases. Inhibition of mTOR promotes triacylglycerol lipolysis and release of free fatty acids, blocks adipogenesis, and impairs the maintenance of fat cells [42-44]. Our data showed that Sirt1 decreased the phosphorylation level of mTOR/S6K1 signal pathway, while Rapamycin, a special inhibitor of mTOR signal significantly 
attenuated this effect. Consistently, we found both Foxos and IL-6 were reduced by Sirt1 treatment and reversed by the addition of Rapamycin. This suggested an association between inflammatory cytokine decrease and mTOR inactivation by the interaction of Sirt1 and Akt/Foxos. In conclusion, our findings demonstrate that Sirt1 decreases adipose inflammation by directly banding with Foxos. Moreover, mTOR/S6K1 signal pathway is essential in the process of inhibiting adipose inflammation. This will promise as a novel strategy for the treatment of obesity-associated inflammation.

\section{Materials and Methods}

\section{Animal Studies}

Eight-week-old BALB/c male mice were purchased from the Vital River Laboratory Animal Technology Company (Beijing, China). All mice experiments were carried out in accordance with the protocol approved by the Animal Ethics Committee and the experimental protocol was performed in accordance with applicable guidelines and regulations. Mice were allowed ad libitum access to water and standard laboratory diet for the first two weeks and kept in the animal room $\left(25^{\circ} \mathrm{C} \pm 1{ }^{\circ} \mathrm{C}\right.$, humidity at $55 \pm 5 \%$, and a $12 \mathrm{~h}$ light/ 12-dark cycle). Mice were subsequently randomly assigned to two groups: high-fat diet fed group (60 Kal\%; Animal Center of the Fourth Military Medical University) or chow diet fed group (10 Kal\%; Animal Center of the Fourth Military Medical University) for the next 6 weeks. Then mice were tail vein injection with Ad-Sirt1 or sh-Sirt1 for the following 7 days. Then animals were euthanized immediately after insulin treatment, subcutaneous adipose tissues were quickly collected in liquid nitrogen and kept at $-80^{\circ} \mathrm{C}$ for subsequent analysis. For LPS $(100 \mu \mathrm{g} / \mathrm{kg}$; Sigma; USA) challenge study, animals were an intraperitoneal injection for 3 days before Ad-Sirt1 or sh-Sirt1 tail vein injection treatment.

\section{Metabolic Phenotyping}

Body weight and food intake were recorded once a week [45]. Visceral fat, epididymal fat and inguinal fat were harvested and weighed. The serum concentrations of interleukin-1 $\beta$ (IL-1 $\beta$ ) and interleukin-6, 4, 10 (IL-6,4,10) were detected using commercial ELISA kits (Cell Signaling Technology, USA) according to the protocol.

\section{Primary Adipocyte Culture}

Inguinal white adipose tissue was harvested, visible fibers and blood vessels were removed, and the adipose tissue was washed three times with PBS buffer containing 200U/mL penicillin (Sigma, St. Louis, USA) and 200U/mL streptomycin (Sigma, St. Louis, USA). Then the adipose tissue was minced into fine sections (1 mm3) with scissors and incubated in $10 \mathrm{~mL}$ of digestion buffer containing Dulbecco's modified Eagle medium (DMEM)/F-12 (Gibco, USA), 100 mM HEPES (Sigma, St. Louis, USA), 1.5\% bovine serum albumin (Sigma, St. Louis, USA), 2 mg/mL type I collagenase (Sigma, St. Louis, USA) for $50 \mathrm{~min}$ at $37^{\circ} \mathrm{C}$ in a water bath. After the incubation, growth medium (DMEM/F-12 (50:50)), 15\% fetal bovine serum (Sigma, St. Louis, USA), $100 \mathrm{U} / \mathrm{mL}$ penicillin and $100 \mathrm{U} / \mathrm{mL}$ streptomycin were added to the digestion flask. Flask contents were mixed and filtered through nylon screens with $250 \mu \mathrm{m}$ and $20 \mu \mathrm{m}$ mesh openings to remove undigested tissue and large cell aggregates. The filtered cells were centrifuged at $1,300 \times \mathrm{g}$ for $7 \mathrm{~min}$ at room temperature to separate floating adipocytes from cell pellets. Finally, cells were seeded into $35-\mathrm{mm}$ primary culture dishes at a density of $8 \times 104$ cells/dish and incubated at $37^{\circ} \mathrm{C}$ under a humidified atmosphere of $5 \% \mathrm{CO}_{2}$ and $95 \%$ air until confluence.

\section{Drug Treatment in Primary Adipocytes}

Primary adipocytes from white adipose tissue were treated with $100 \mathrm{ng} / \mathrm{ml}$ LPS for $12 \mathrm{~h}$ to stimulate adipose inflammation. At the end of the treatment, adipocytes were collected for further detection. Ad-Foxo1 and Ad-Foxo3a and Ad-Sirt1-mutant recombinant adenovirus vectors were kept in our lab. Cell viability was measured by CCK-8 Lazy me, China) assay. Collected cells were seeded in 96-well plate at a density of $5 \times 103$ and cultured for 12 h. $10 \mu$ l CCK- 8 was added into each well and incubated for 1 hour at $37^{\circ} \mathrm{C}$. Absorbance was quantified at $450 \mathrm{~nm}$ by Vector 5 (Bio-Tech Instruments, USA). In signal transduction events, cells were pretransfected with Ad-Sirt1 or sh-Sirt1 and then incubated with $5 \mathrm{nM}$ Rapamycin for $60 \mathrm{~min}$.

\section{Immunoprecipitation (IP) and Chromatin Immunopre- cipitation (ChIP) Analyse}

Primary adipocytes were transfected with plasmids include His-Sirt, Akt, His-Akt, Sirt1, Foxo1, His-Foxo1, Foxo3a and HisFoxo3a. The whole cell lysate was harvested in lysis buffer $48 \mathrm{~h}$ post-transfection. The supernatant of whole cell lysate was precleared with protein A for $2 \mathrm{~h}$ and incubated with $4 \mu \mathrm{g}$ primary antibody overnight at $4^{\circ} \mathrm{C}$. Immune complexes were pulled down with protein $\mathrm{A}$ for $2 \mathrm{~h}$ at $4^{\circ} \mathrm{C}$ with shaking. Beads were washed once with lysis buffer and three times with wash buffer followed by western blot analysis.

Adipocytes were prepared for Chromatin Immunoprecipitation (ChIP) assay using a ChIP assay kit (Abcam, Cambridge, UK) according to the manufacturer's protocol. Primary antibodies of Foxo1 and Foxo3a (ab39670 and ab12162, Abcam, Cambridge, UK) or IgG (ab171870, Abcam, Cambridge, UK) were used. DNA-protein crosslinking complexes were collected, and purified DNA was subjected to qPCR with SYBR green fluorescent dye (Invitrogen, Californian, USA)

\section{Real-Time PCR Analysis}

Total RNA was extracted with Tripura Reagent kit (Takara, Dalian, China) and 400ng of total RNA was reverse transcribed using the M-MLV reverse transcriptase kit (Takara, China). Primers for the study were synthesized by Shanghai Sangon Ltd. (Shanghai, China). Quantitative PCR was performed in $25 \mu \mathrm{L}$ reactions containing specific primers and SYBR Premix EX Taq (Takara, Dalian, China). The levels of mRNAs were normalized to $\beta$-actin. The expression of genes was analyzed by the method of $2-\Delta \triangle \mathrm{Ct}$. 


\section{Protein Extraction and Western Blot Analysis}

Cells were lysed in RIPA buffer for $40 \mathrm{~min}$ at $4^{\circ} \mathrm{C}$. Removed insoluble material by centrifugation at $12,000 \times \mathrm{g}$ for $15 \mathrm{~min}$ at $4^{\circ} \mathrm{C}$, and the supernatants were used to assay protein levels. Protein samples $(50 \mu \mathrm{g})$ were separated by electrophoresis on $12 \%$ and 5\% SDS-PAGE gels using slab gel apparatus and then transferred to PVDF nitrocellulose membranes (Millipore, USA) blocked with $5 \%$ skim milk powder/Tween 20/TBST at room temperature for 2 h. Primary antibodies against Raptor, p-mTORSer2448, mTORSer2448, p-S6K1Thr389, S6K1Thr389 p-Akt, Akt and GAPDH were purchased from Bioworld (USA). Antibodies against Sirt1, leptin, IL-6, MCP-1, TNF- $\alpha$, and Foxo1, Foxo3a, target-His were formed Abcam (Cambridge, UK). Rapamycin (Rapa) was from Sigma (USA). Membranes were incubated with primary antibodies at $4^{\circ} \mathrm{C}$ overnight and then incubated with the appropriate HRP-conjugated secondary antibodies (Baoshan, China) for $2 \mathrm{~h}$ at room temperature. Proteins were visualized using chemiluminescent peroxidase substrate (Millipore, USA), and then the blots were quantified using ChemiDoc XRS system (Bio-Rad, USA) and Quantitative analysis of immune-blotted bands was performed using Quantity One software (Bio-Rad, USA).

\section{Statistical Analysis}

Statistical analyses were conducted using SAS v8.0 (SAS Institute, Cary, NC). Data were analyzed using one-way ANOVA. Comparisons among individual means were made by Fisher's Least Significant Difference (LSD). Data were presented as mean \pm SD. $p<$ 0.05 was considered to be statistically significant.

\section{Acknowledgment}

This work was supported by a grant from Tibet Science and Technology Key Science and Technology Project.

\section{References}

1. Rodgers JT, Lerin C, Haas W, Gygi SP, Spiegelman BM, et al. (2005) Nutrient control of glucose homeostasis through a complex of PGC- $1 \alpha$ and SIRT1. Nature 434: 113-118.

2. Picard F, Kurtev M, Chung N, Topark Ngarm A, Senawong T, et al. (2004) Guarente, Sirt1 promotes fat mobilization in white adipocytes by repressing PPAR- $\gamma$. Nature 429: 771-776.

3. Yoshizaki T, Milne JC, Imamura T, Schenk S, Sonoda N, et al. (2009) SIRT1 exerts anti-inflammatory effects and improves insulin sensitivity in adipocytes. Mol Cell Biol 29: 1363-1374.

4. Hotamisligil GS, Arner P, Caro JF, Atkinson RL, Spiegelman BM, et al. (1995) Increased adipose tissue expression of tumor necrosis factoralpha in human obesity and insulin resistance. J Clin Invest 95(5): 24092415.

5. Matthew P Gillum, Maya E Kotas, Derek M Erion, Romy Kursawe, Paula Chatterjee, et al. (2011) SirT1 regulates adipose tissue inflammation. Diabetes 60: 3235-3245.

6. Guarente L (2006) Sirtuins as potential targets for metabolic syndrome. Nature 444: 868-874.

7. Takeshi Yoshizaki, Simon Schenk, Takeshi Imamura, Jennie L Babendure, Noriyuki Sonoda, et al. (2010) SIRT1 inhibits inflammatory pathways in macrophages and modulates insulin sensitivity. Am J Physiol Endocrinol Metab 298: E419-E428.

8. Purushotham A, Schug TT, Xu Q, Surapureddi S, Guo X, et al. (2009) Hepatocyte-specific deletion of SIRT1 alters fatty acid metabolism and results in hepatic steatosis and inflammation. Cell Metab 9: 327-338.

9. Luo J, Nikolaev AY, Imai S, Chen D, Su F (2001) Negative control of p53 by Sir2 $\alpha$ promotes cell survival under stress. Cell 107: 137-148.

10. Martel RR, Botros IW, Rounseville MP, Hinton JP, Staples RR, et al. (2002) Multiplexed screening assay for mRNA combining nuclease protection with luminescent array detection. Assay Drug Dev Technol 1: 61-71.

11. Cinti S, Mitchell G, Barbatelli G, Murano I, Ceresi E, et al. (2005) Adipocyte death defines macrophage localization and function in adipose tissue of obese mice and humans. J Lipid Res 46: 2347-2355.

12. Ozcan U, Cao Q Yilmaz E, Lee AH, Iwakoshi NN, et al. (2004) Endoplasmic reticulum stress links obesity, insulin action, and type 2 diabetes. Science 306: 457-461.

13. Hosogai N, Fukuhara A, Oshima K, Miyata Y, Tanaka S, et al. (2007) Adipose tissue hypoxia in obesity and its impact on adipocytokine dysregulation. Diabetes 56: 901-911.

14. Nakae J, Kitamura T, Kitamura Y, Biggs WH, Arden KC, et al. (2003) The forkhead transcription factor Foxo1 regulates adipocyte differentiation. Developmental cell 4: 119-129.

15. Gross DN, Van Den Heuvel AP, Birnbaum MJ (2008) The role of FoxO in the regulation of metabolism. Oncogene 27: 2320-2336.

16. Nakae J, Cao Y, Oki M, Orba Y, Sawa H, et al. (2008) Forkhead transcription factor Fox01 in adipose tissue regulates energy storage and expenditure. Diabetes 57: 563-576.

17. Kajno E, Mc Graw TE, Gonzalez E (2015) Development of a new model system to dissect isoform-specific Akt signaling in adipocytes. Biochemical Journal 468: 425-434.

18. Cohen S, Lee D, Zhai B, Gygi SP, Goldberg AL (2014) Trim32 reduces PI3K-Akt-FoxO signaling in muscle atrophy by promoting plakoglobinPI3K dissociation. J Cell Biol 204: 747-758.

19. Frescas D, Valenti L, Accili D (2005) Nuclear trapping of the forkhead transcription factor Fox01 via Sirt-dependent deacetylation promotes expression of glucogenetic genes. J Biol Chem 280: 20589-20595.

20. Bodine SC, Stitt TN, Gonzalez M, Kline WO, Stover GL, et al. (2001) Akt/ mTOR pathway is a crucial regulator of skeletal muscle hypertrophy and can prevent muscle atrophy in vivo. Nat Cell Biol 3: 1014-1019.

21. Liu M, Bai J, He S, Villarreal R, Hu D, et al. (2014) Grb10 promotes lipolysis and thermogenesis by phosphorylation-dependent feedback inhibition of mTORC1. Cell Metab 19: 967-980.

22. Guertin DA, Sabatini DM (2007) Defining the role of mTOR in cancer Cancer cell 12: 9-22.

23. Sarbassov DD, Guertin DA, Ali SM, Sabatini DM (2005) Phosphorylation and regulation of Akt/PKB by the rictor-mTOR complex. Science 307: 1098-1101.

24. Ravikumar B, Vacher C, Berger Z, Davies JE, Luo S, et al. (2004) Inhibition of mTOR induces autophagy and reduces the toxicity of polyglutamine expansions in fly and mouse models of Huntington disease. Nature Genetics 36: 585-595.

25. Khamzina L, Veilleux A, Bergeron S, Marette A (2005) Increased activation of the mammalian target of rapamycin pathway in liver and skeletal muscle of obese rats: Possible involvement in obesity-linked insulin resistance. Endocrinology 146: 1473-1481.

26. Declercq IA, Da Silva Morais A, Schroyen B, Van Hul N, Geerts A (2007) Insulin resistance in hepatocytes and sinusoidal liver cells: Mechanisms and consequences. Journal of Hepatology 47: 142-156. 
27. Jiang H, Westerberg M, Wang C, Zhu Y, Ai D (2014) Macrophage mTORC1 disruption reduces inflammation and insulin resistance in obese mice. Diabetologia 57: 2393-2404.

28. Busch F, Mobasheri A, Shayan P, Stahlmann R, Shakibaei M (2012) Sirt-1 is required for the inhibition of apoptosis and inflammatory responses in human tenocytes. Journal of Biological Chemistry 287: 25770-25781.

29. Arkan MC, Hevener AL, Greten FR, Maeda S, Li ZW, et al. (2005) IKK- $\beta$ links inflammation to obesity-induced insulin resistance. Nature medicine 11: 191-198.

30. Hotamisligil GS (2006) Inflammation, and metabolic disorders. Nature 444: 860-867.

31. Lagouge M, Argmann C, Gerhart Hines Z, Meziane H, Lerin C, et al. (2006) Resveratrol improves mitochondrial function and protects against metabolic disease by activating SIRT1 and PGC-1 $\alpha$. Cell 127: 1109-1122.

32. Milne JC, Lambert PD, Schenk S, Carney DP, Smith JJ, et al. (2007) Small molecule activators of SIRT1 as therapeutics for the treatment of type 2 diabetes. Nature 450: 712-716.

33. Seiler F, Hellberg J, Lepper PM, Kamyschnikow A, Herr C, et al. (2013) FOXO transcription factors regulate innate immune mechanisms in respiratory epithelial cells. The Journal of Immunology 190: 1603-1613.

34. Salminen A, Ojala J, Huuskonen J, Kauppinen A, Suuronen T, et al. (2008) Interaction of aging-associated signaling cascades: inhibition of NF- $\kappa \mathrm{B}$ signaling by longevity factors FoxOs and SIRT1. Cellular and Molecular Life Sciences 65: 1049-1058.

35. Kadowaki T, Ueki K, Yamauchi T, Kubota N (2012) Snap Shot: Insulin signaling pathways. Cell 148: 624-624. e621.

36. Kidd LB, Schabbauer GA, Luyendyk JP, Holscher TD, Tilley RE, et al. (2008) Insulin activation of the phosphatidylinositol 3-kinase/protein kinase $\mathrm{B}$ (Akt) pathway reduces lipopolysaccharide-induced inflammation in

\section{ISSN: 2574-1241}

DOI: 10.26717/BJSTR.2019.19.003371

Bin Li. Biomed J Sci \& Tech Res

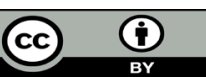

This work is licensed under Creative

Commons Attribution 4.0 License

Submission Link: https://biomedres.us/submit-manuscript.php mice. Journal of Pharmacology and Experimental Therapeutics 326: 348-353.

37. Cohen HY, Miller C, Bitterman KJ, Wall NR, Hekking B, et al (2004) Calorie restriction promotes mammalian cell survival by inducing the SIRT1 deacetylase. Science 305: 390-392.

38. Nisoli E, Tonello C, Cardile A, Cozzi V, Bracale R Clementi, et al. (2005) Calorie restriction promotes mitochondrial biogenesis by inducing the expression of eNOS. Science 310: 314-317

39. Pang W, Wang Y, Wei N, Xu R, Xiong Y, et al. (2013) Sirt1 inhibits akt2mediated porcine adipogenesis potentially by direct protein-protein interaction. PloS one 8: e71576.

40. Lee DF, Kuo HP, Chen CT, Hsu JM, Chou CK, et al. (2007) IKK $\beta$ suppression of TSC1 links inflammation and tumor angiogenesis via the mTOR pathway. Cell 130: 440-455.

41. Greten FR, Eckmann L, Greten TF, Park JM, Li ZW, et al. (2004) IKK $\beta$ links inflammation and tumorigenesis in a mouse model of colitis-associated cancer. Cell 118: 285-296.

42. Tremblay F, Gagnon A, Veilleux A, Sorisky A, Marette A (2005) Activation of the mammalian target of rapamycin pathway acutely inhibits insulin signaling to Akt and glucose transport in 3T3-L1 and human adipocytes. Endocrinology 146: 1328-1337.

43. Chakrabarti P, English T, Shi J, Smas CM, Kandror KV (2010) Mammalian target of rapamycin complex 1 suppresses lipolysis, stimulates lipogenesis, and promotes fat storage. Diabetes 59: 775-781.

44. Soliman GA (2011) The integral role of mTOR in lipid metabolism. Cell Cycle 10: 861-862.

45. Lee MO (1929) Determination of the surface area of the white rat with its application to the expression of metabolic results. American Journal of Physiology--Legacy Content 89: 24-33.

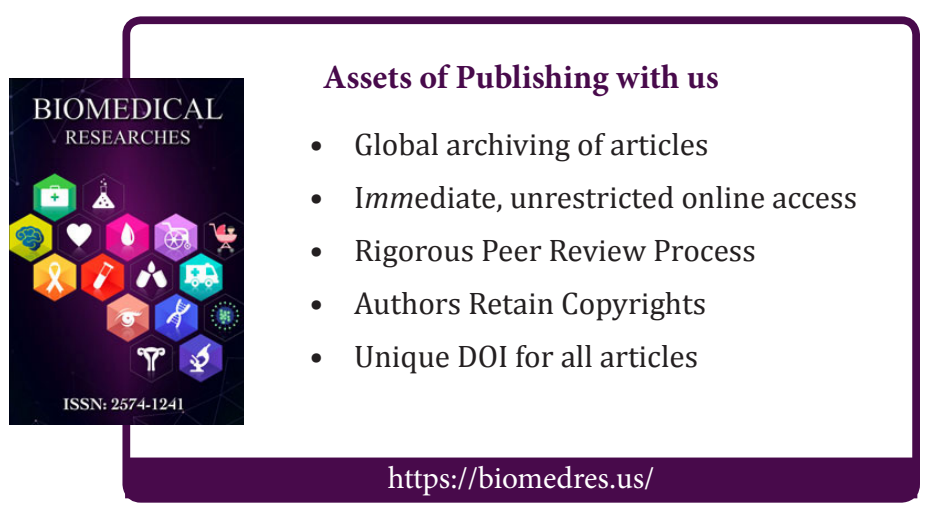

